Bol. Soc. Bot. México 59: 99-113 (1996)

\title{
PhyLOGenetic ANALYSIS OF
}

\section{LARGE MOLECULAR DATA SETS}

\author{
Pamela S. Soltis y Douglas E. Soltis \\ Department of Botany, Washington State University, Pullman, WA 99164-4238, USA.
}

\begin{abstract}
Resumen. Los adelantos tecnológicos en biología molecular han aumentado en gran medida la velocidad y eficiencia de la secuenciación de $\mathrm{ADN}$, haciendo posible construir relativamente rápido grandes juegos de datos moleculares para la reconstrucción filogenética. A pesar de su potencial para mejorar nuestro entendimiento de la filogenia, estos grandes juegos de datos también proveen muchos retos. En este artículo discutimos varios de estos desafíos, incluyendo 1] el fracaso en la búsqueda del árbol más parsimonioso (el óptimo local) en un lapso de tiempo razonable, 2] la diferencia entre un óptimo local y el óptimo global, y 3] la existencia de clases múltiples (islas) de árboles más parsimoniosos. También discutimos posibles estrategias para aumentar la posibilidad de encontrar el o los árboles más parsimoniosos y presentamos dos ejemplos de nuestro trabajo en la filogenia de las angiospermas. Concluimos con una discusión de dos alternativas para el análisis de los juegos enteros de datos: el "enfoque de representantes" y la "compartamentalización". Sugerimos que debe darse consideración adicional al asunto del análisis de juegos grandes de datos, sean morfológicos o moleculares.

Palabras clave: óptimo local, óptimo global, islas, enfoque de representantes, compartamentalización.

Abstract. Technological advances in molecular biology have greatly increased the speed and efficiency of DNA sequencing, making it possible to construct large molecular data sets for phylogeny reconstruction relatively quickly. Despite their potential for improving our understanding of phylogeny, these large data sets also provide many challenges. In this paper, we discuss several of these challenges, including 1] the failure of a search to find the most parsimonious trees (the local optimum) in a reasonable amount of time, 2] the difference between a local optimum and the global optimum, and 3] the existence of multiple classes (islands) of most parsimonious trees. We also discuss possible strategies to improve the likelihood of finding the most parsimonious tree(s) and present two examples from our work on angiosperm phylogeny. We conclude with a discussion of two alternatives to analyses of entire large data sets, the exemplar approach and compartmentalization, and suggest that additional consideration must be given to issues of data analysis for large data sets, whether morphological or molecular.
\end{abstract}

Key words: local optimum, global optimum, islands, exemplar approach, compartmentalization.

\begin{abstract}
A dvances in molecular biology have greatly improved the speed and efficiency of DNA sequencing, making it possible to generate large, comprehensive data sets for phylogenetic analysis in a relatively short period of time. This ability to amass large molecular data sets has provided a new set of challenges for molecular systematists: how, in fact, does one conduct a phylogenetic analysis of several thousand characters for several hundred taxa and be even moderately confident in the results? The scope of such analyses goes far beyond anything faced in phylogenetic analysis of other characters, such as morphology and chemis-
\end{abstract}

try, and theoreticians and molecular phylogeneticists are only now beginning to identify the problems that arise in such analyses and to recommend solutions to these problems. In this paper, we will 1] review some of the challenges provided by large data sets, whether they are morphological or molecular, 2] offer some possible strategies for analyzing large data sets, and $3]$ provide two examples of phylogenetic analyses of large molecular data sets for angiosperms.

Problems posed by large data sets. Exact parsimony-based analyses that are, by definition, guaranteed to find the shortest trees are computationally feasible only for 


\begin{tabular}{|rl|}
\hline $\begin{array}{l}\text { Table 1. The number of rooted evolutionary trees } \\
\text { for } n \text { taxa (from Felsenstein, 1978a) }\end{array}$ \\
\hline$n$ & Number of Trees \\
\hline & \\
1 & 1 \\
2 & 1 \\
3 & 4 \\
4 & 26 \\
5 & 236 \\
6 & 2,752 \\
7 & 39,208 \\
8 & 660,032 \\
9 & $12,818,912$ \\
10 & $282,137,824$ \\
15 & $6,353,726,042,486,112$ \\
20 & $887,094,711,304,094,583,095,296$ \\
\hline
\end{tabular}

small data sets. For example, exhaustive searches that compare all possible trees are generally not possible for data sets containing more than about 10 taxa, for which there are $282,137,824$ possible rooted trees (table 1; Felsenstein, 1978a). The branch-and-bound algorithm (Hendy and Penny, 1982) and its recent modification (Swofford and Olsen, 1990) are only feasible for data sets with as many as 20-25 taxa. Even with recent improvements in the computational speed of maximum likelihood approaches (Felsenstein, 1981) to phylogeny reconstruction (e.g., as in PAUP* 4.0; Swofford, pers. comm.), maximum likelihood cannot realistically be applied to data sets of more than perhaps 70-100 taxa. Because the number of possible trees increases exponentially with the number of taxa (Felsenstein, 1978a), data sets with larger numbers of taxa can only be analyzed with heuristic searches.
As approximations, heuristic searches have the inherent risk that maximum parsimony will not be realized. Efforts to reduce this risk are computationally expensive, and the systematist must balance the risk of missing the shortest tree(s) with the costs of a thorough, and prolonged, heuristic search. A heuristic search is intended to find the most parsimonious tree(s), and it may or may not be successful. The analogy of hill-climbing is often applied to a heuristic search ( $c f$. Swofford and Olsen, 1990; figure 1). The search for shorter trees is analogous to climbing a hill, where the peak is equated with maximum parsimony (figure 1a). Various search strategies may improve one's likelihood of reaching the peak and one's efficiency in doing so. With small data sets, this peak is typically considered to represent the best estimate of phylogeny. However, one's search may have reached its peak, but it was ascending the wrong mountain (figure 1b). That is, shorter trees than those accessible from the beginning hill exist atop other mountains. This difference between local optimum (top of the hill climbed) and global optimum (peak of tallest mountain) has long been recognized. The likelihood of inadvertantly and unknowingly reaching a local optimum rather than a global optimum increases with large data sets, and search strategies must be designed to avoid inferring maximum parsimony from only a local optimum. Furthermore, with large data sets it may be difficult to achieve even a local optimum because branch-swapping on a huge tree is computationally intensive, taking a very long time and generating a large number of additional trees.

As Maddison (1991) pointed out, even if one attains a global optimum, there may be multiple peaks of equally parsimonious trees (figure 1c). Therefore, climbing a single hill will give only a subset of all

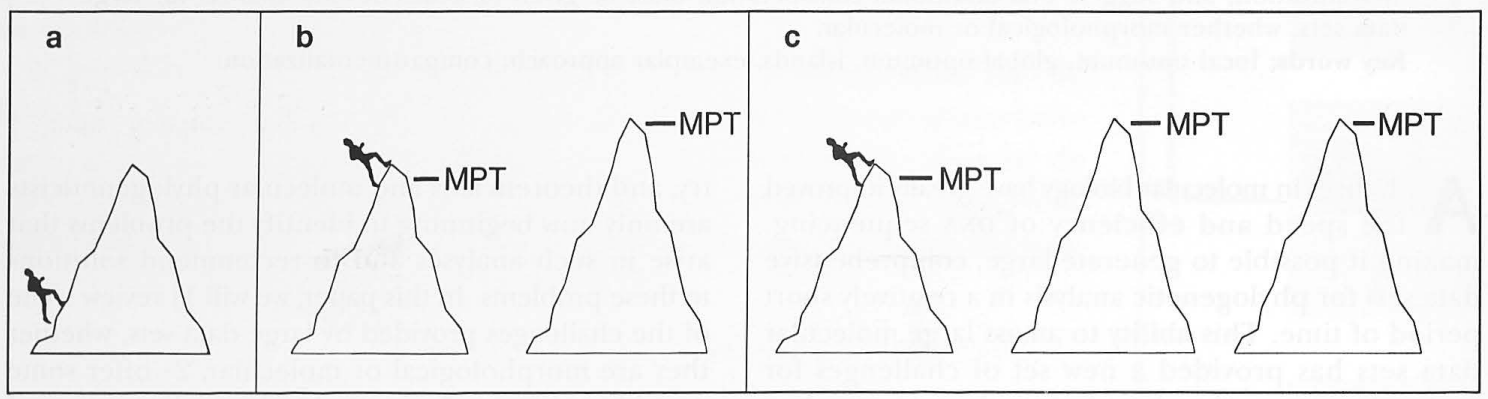

Figure 1. The "hill-climbing" analogy for the search for most parsimonious trees (MPT) (after Swofford and Olsen, 1990). 1a. The climb up a single hill, where the peak is equated with maximum parsimony. 1b. The local optimum (peak of hill climbed) may differ from the global optimum (peak of tallest mountain), meaning shorter trees than those recovered exist atop other mountains. 1c. Multiple peaks (groups) of MPT; climbing a single hill will reveal only a subset of all MPT. 
possible trees while other groups of trees go undiscovered. Each group of trees obtained from a single peak will be more closely related to each other than to groups from other peaks. Because trees on different peaks represent groups of trees that differ from each other by two or more rearrangements, it is not possible to travel from one peak to another. For this reason, Maddison (1991) used the metaphor of islands of most parsimonious trees (figure 2). Heuristic analyses also need to search for multiple islands and provide mechanisms to prevent a search from becoming marooned on a single island.

Given the possibilities described above, a heuristic search of a large data set may never reach a local optimum. Even if it does, this local optimum may not be the global optimum; additional groups of trees of equal or shorter length may remain undiscovered. Given the very real possibility of finding neither the minimal tree(s) nor all groups of minimal trees in a heuristic search, what tree-searching strategies can be used to improve one's ability to detect most, if not all, minimal trees?

\section{Phylogenetic analysis of large data sets}

Heuristic search strategies for large data sets. Because heuristic searches are not guaranteed to find the shortest tree(s), searches should be designed to explore as much of the "tree space" (i.e., the realm of all possible trees) as possible to improve the likelihood of finding the global optimum (or optima), that is, the shortest trees (figure 3). A single search, which essentially enters tree space from a single location, will likely not find the shortest tree(s). Additional tree space can be explored through multiple searches, each starting with a different tree and thus entering tree space from a different location (figure 3 ). Biases reflected in the trees saved by each search will most likely be offset by pooling the results of multiple searches. In practice, these multiple searches can be performed by using random addition of taxa (in PAUP, Swofford, 1991; the jumble option in PHYLIP, J. Felsenstein) to provide different starting points for the exploration of tree space. Multiple searches will not only improve the likelihood of finding a global optimum (or opti-

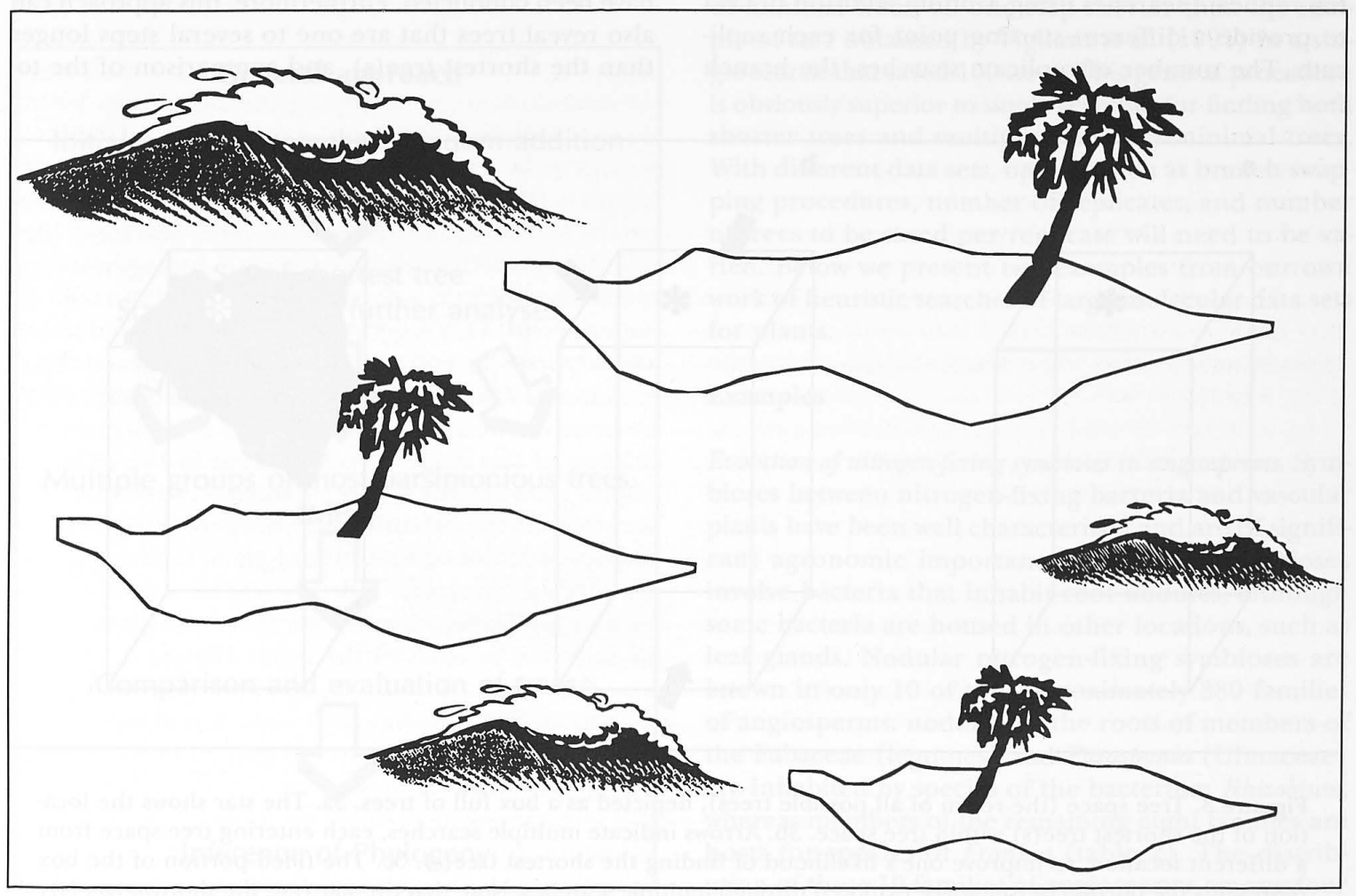

Figure 2. Islands of MPT (sensu Maddison, 1991). Trees on different islands represent groups of trees that differ from each other by at least two or more rearrangements, making it impossible to travel from one island to another. 
$\mathrm{ma})$, but they will also likely discover additional groups of most parsimonious trees ( $c f$. Maddison, 1991; Maddison et al., 1992). Of course, performing 100 or more replicate searches will increase computation time substantially, and it may be necessary with very large data sets to save only a subset of the trees recovered per replicate or to use less thorough branch swapping procedures (such as nearest neighbor interchange (NNI) rather than tree bisection reconnection (TBR)). Although both of these options limit the potential of any individual search to find the shortest trees, the results of an increased number of replicate searches will likely offset the inadequacies of the individual searches.

Because each data set offers its own challenges, no formula can be followed to ensure that heuristic searches will find the shortest trees (Swofford and Olsen, 1990). However, the primary goal of the search approach should be to explore as much of tree space as possible. We offer the following guidelines to increase the likelihood that analyses of large molecular data sets will find some, if not all, of the shortest trees (summarized in figure 4 ). We recommend initial replicate searches using random addition of taxa to provide a different starting point for each replicate. The number of replicate searches, the branch swapping procedure, and the number of trees saved per replicate will depend on the size of the data set. The use of a more thorough branch swapping procedure such as TBR, along with increases in the number of replicates and the number of trees saved per replicate, will improve the likelihood of ultimately finding the shortest trees. However, these options may not be feasible with all data sets; the goal of the analysis should remain the exploration of as much of tree space as possible. The shortest trees obtained through these initial, often coarse, searches can then be used as starting trees for more thorough searches that use TBR branch swapping and save all most parsimonious trees (MPT). This approach will allow the recovery of multiple classes of MPT, and these trees can be compared, evaluated, and used to infer relationships. Of course, it is possible that the optimal trees cannot be reached from those trees used as starting trees; it may therefore also be valuable to use slightly suboptimal trees from the initial searches as starting trees in subsequent searches. The shortest trees obtained by this general procedure may therefore not represent the global optimum, but a valiant exploration of tree space will have been conducted. Furthermore, this approach can also reveal trees that are one to several steps longer than the shortest tree(s), and comparison of the to- a

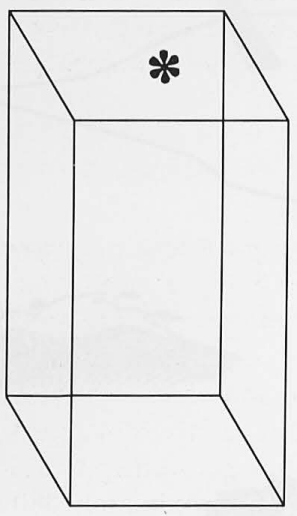

b

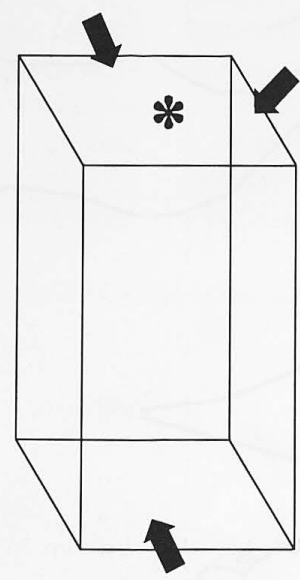

C

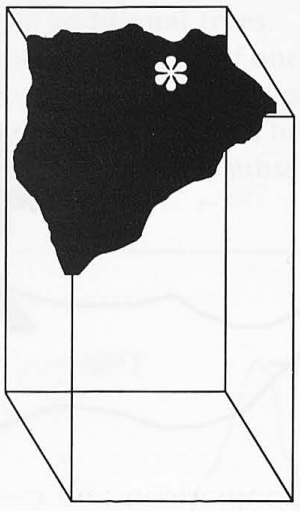

Figuure 3. Tree space (the realm of all possible trees), depicted as a box full of trees. 3a. The star shows the location of the shortest tree(s) within tree space. $3 \mathrm{~b}$. Arrows indicate multiple searches, each entering tree space from a different location, to improve one's likelihood of finding the shortest tree(s). 3c. The filled portion of the box represents the portion of tree space explored through multiple searches. Note that the star (i.e., the shortest tree(s)) was discovered by this search, but that a large portion of tree space remained unexplored. Had the shortest tree(s) been located in the unfilled portion of the box, the search would not have recovered the shortest trees; that is, a local optimum may have been reached, but not the global optimum. 
Table 2. Angiosperm families that have one or more species known to engage in symbiotic associations with nitrogen-fixing bacteria in root nodules. The proportion of genera in each family with root nodules and the genus of bacterial symbiont for each family are given. Estimates of the total number of genera for each family are taken from Cronquist (1981); data on nodule formation are from Akkermans and van Dijk (1981) and Torrey and Berg (1988).

\begin{tabular}{lll}
\hline $\begin{array}{l}\text { Angiosperm } \\
\text { Family }\end{array}$ & Proportion & $\begin{array}{l}\text { Bacterial } \\
\text { Symbiont }\end{array}$ \\
\hline Betulaceae & $1 / 6$ & Frankia \\
Casuarinaceae & $4 / 4$ & Frankia \\
Coriariaceae & $1 / 1$ & Frankia \\
Datiscaceae & $1 / 3$ & Frankia \\
Elaeagnaceae & $3 / 3$ & Frankia \\
Fabaceae & most examined/640 & Rhizobium \\
Myricaceae & $2 / 3$ & Frankia \\
Rhamnaceae & $7 / 55$ & Frankia \\
Rosaceae & $5 / 100$ & Frankia \\
Ulmaceae & $1 / 18$ & Rhizobium \\
\hline
\end{tabular}

\section{General approach}

Initial replicate searches: random addition

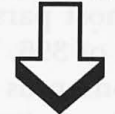

Set of shortest tree

Starting trees for further analyses
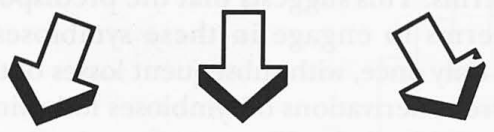

Multiple groups of most parsimonious trees

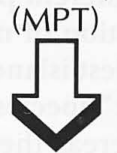

Comparison and evaluation of trees

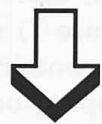

Inference of Phylogeny

Figure 4. Flow chart showing a general approach to the analysis of large data sets. pologies of the shortest trees with those of slightly longer ones can provide a rough measure of the robustness of the topology of the MPT, similar to a decay analysis (Bremer, 1988; Donoghue et al., 1992). From the pragmatic perspective, this general approach has proven computationally feasible for data sets of 75-100 taxa and $+/-1,400$ base pairs (bp), providing pools of MPT in 2-6 months. Obviously, data sets of this size preclude detailed analyses of internal branch support such as bootstrap (Felsenstein, 1985) or decay analyses ( $c f$. Maddison et al., 1992).

Larger data sets (i.e., > 100 taxa) may require additional constraints to prevent individual searches from being overburdened with swapping on suboptimal trees. Maddison et al. (1992) present a search strategy for data sets of this size. They performed initial replicate searches of 149 human mtDNA sequences (data from Vigilant et al., 1991) using random addition of taxa and TBR branch swapping and saving only two trees per search (by setting PAUP's options NCHUCK $=2$ and CHUCKLEN $=2$ ). The trees obtained from these replicates were subsequently used as starting trees for further searches. This approach found many thousands of minimal trees, all one step shorter than the sample of MPT obtained by Vigilant et al. (1991) in a single search that saved 100 trees. This general procedure is obviously superior to single searches for finding both shorter trees and multiple classes of minimal trees. With different data sets, options such as branch swapping procedures, number of replicates, and number of trees to be saved per replicate will need to be varied. Below we present two examples from our own work of heuristic searches of large molecular data sets for plants.

\section{Examples}

Evolution of nitrogen-fixing symbioses in angiosperms. Symbioses between nitrogen-fixing bacteria and vascular plants have been well characterized and are of significant agronomic importance. Most such symbioses involve bacteria that inhabit root nodules, although some bacteria are housed in other locations, such as leaf glands. Nodular nitrogen-fixing symbioses are known in only 10 of the approximately 380 families of angiosperms: nodules on the roots of members of the Fabaceae (legumes) and Parasponia (Ulmaceae) are inhabited by species of the bacterium Rhizobium, whereas members of the remaining eight families are hosts for species of Frankia (table 2). The distribution of these 10 families of angiosperms among four of Cronquist's (1981) six subclasses of dicots (figure 5 ) has been considered evidence that the ability to form symbioses with nitrogen-fixing bacteria has 


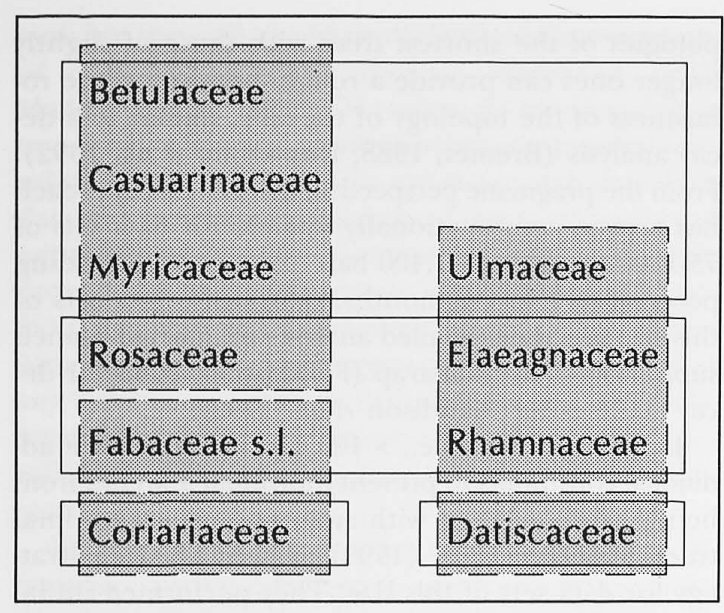

Figure 5. Phylogenetic relationships among angiosperm families known to have symbiotic associations with nitrogen-fixing bacteria, as inferred from the classifications of Cronquist (1981) and Dahlgren (1980). Families classified together are placed together within a box; solid lines represent Cronquist's (1981) subclasses, and dashed lines represent Dahlgren's (1980) superorders.

evolved multiple times in very different lineages of angiosperms (Baker and Mullin, 1992; Mullin et al., 1990). This hypothesis has significant agronomic implications: if the hosts are as phylogenetically distant as current classifications suggest, then the bacteria have been able to adapt to a broad range of genetic backgrounds and could perhaps be "coaxed" to nodulate and inhabit new hosts, such as crop species that do not naturally enter into such symbioses (Sprent and Raven, 1992; Mullin, 1992; Al-Mallah et al., 1989).

In contrast to traditional classifications of angiosperms (e.g., Cronquist, 1981; Takhtajan, 1980; Thorne, 1992; Dahlgren, 1980), phylogenetic analyses of $r b c \mathrm{~L}$ sequences for 499 species of seed plants (Chase et al., 1993) suggested that representatives of angiosperm families that engage in nodular nitrogenfixing symbioses occur as part of a single clade (termed "Rosid I" by Chase et al., 1993, because it comprises primarily members of Rosidae), along with several families that do not participate in these symbioses (figure 6). (This large $r b c \mathrm{~L}$ analysis presents problems of its own, as discussed by Chase et al. (1993) and Rice et al. (1997).) However, the large $r b c \mathrm{~L}$ analysis did not include representatives of all families known to have nitrogen-fixing symbioses. To test the hypothesis that these families with nitrogen-fixing symbioses are in fact much more closely related than current classifications suggest, we performed more detailed analy- ses of the Rosid I clade, with representatives of all 10 families that engage in nitrogen-fixing symbioses and many families that do not (Soltis et al., 1995). The results of this analysis do in fact support the hypothesis of a single origin of the "predisposition" for nitrogen-fixing symbioses; however, it is the methodology rather than the result that is relevant to the present paper.

The analysis of Rosid I (Soltis et al., 1995) included $r b c \mathrm{~L}$ sequences (1,428 bp in length) for 99 species, including five outgroups previously shown to fall well outside of Rosid I (Chase et al., 1993). Heuristic searches were conducted with PAUP 3.1.1 (Swofford, 1991). Given the size of this data set, a single heuristic search will likely not find the most parsimonious tree(s) ( $c f$. Maddison et al., 1992). We therefore adopted the strategy of multiple searches, both to enhance our chances of finding the global optimum and to uncover multiple classes of most parsimonious trees (Maddison, 1991). We conducted 800 replicate searches with random taxon addition and TBR branch swapping; a single shortest tree from each replicate was saved as a possible starting point for subsequent searches. The 24 shortest of these trees (length $=3,559$ steps) were used as starting trees in analyses that used TBR branch swapping to swap to completion and saved all most parsimonious trees. Swapping on these trees led to trees of lengths 3,552-3,559 steps and revealed three groups (islands) of most parsimonious trees (MPT; length $=3,552$ steps): of 396,144 , and 18 trees, respectively. The strict consensus of these 558 trees (figure 7) shows that all species with nitrogen-fixing symbioses fall into a single, relatively small clade of angiosperms. This suggests that the predisposition for angiosperms to engage in these symbioses evolved perhaps only once, with subsequent losses of this ability or recurrent derivations of symbioses following diversification of this clade. However, trees in the different islands show slightly different patterns of relationships and thus of the evolution of nitrogen-fixing symbioses. Trees of the smallest island place the four clades containing "symbiotic" species (clades A-D, figure 7) in a single clade, whereas the strict consensus trees of the trees in each of the other islands do not reveal this clade. Instead, these consensus trees (and that of all 558 MPT; figure 7) show a polytomy of six lineages, four of which contain species with nitrogenfixing symbioses. Swapping on the 24 starting trees was conducted in parallel by three of the collaborators on this project; two analyses used TBR branch swapping, and the third used NNI branch swapping. It is instructive that, whereas both analyses using TBR discovered three islands of MPT, the analysis using NNI failed to locate the island of 18 trees. 
This difference in the numbers and topologies of trees obtained by swapping on different starting trees and by using different methods of branch swapping produces biologically different results. For example, had a more limited search been conducted, we might have recovered only members of the 18-tree island and concluded that those angiosperms that engage in nitrogen-fixing symbioses form a single clade, to the exclusion of two other clades of mostly dilleniids (clades $\mathrm{X}$ and $\mathrm{Y}$, figure 7 ). We might then have inferred an even closer relationship among species with nitrogen-fixing symbioses than this data set warrants and a more recent evolutionary origin of nodular nitrogen-fixing symbioses. Instead, although the multiple searches place the "symbiotic" species in a single, fairly small clade within the angiosperms, we cannot infer this closer relationship among lineages A-D revealed by the trees on the smallest island, even though $74 \%$ of all 558 MPT also show these four lineages forming a single clade. The data support a single evolutionary origin of the ability to engage in nitro- gen-fixing symbioses with nodule-inhabiting bacteria, with subsequent loss of this ability and/or recurrent derivation of symbioses during the diversification of this clade (Soltis et al., 1995). However, although our searches identified multiple groups of MPT and found shorter trees than a single heuristic search recovered (data not shown), it is possible, perhaps even likely, that additional trees of equal or shorter length exist but were not found. Any conclusions drawn from an analysis of a data set of this size must be tempered by the sobering realization that unrecovered trees may conflict with the trees obtained. However, recent analyses of $18 \mathrm{~S}$ rDNA sequences for angiosperms also support a clade similar in composition to the "nitrogenfixing clade" in the $r b c \mathrm{~L}$ analyses (Soltis $e t$ al., 1997). Phylogeny of angiosperms: analysis of $18 S$ rDNA sequences. Recent analyses of both morphological and molecular data have greatly improved our understanding of angiosperm phylogeny(e.g., Donoghue and Doyle, 1989a, 1989b; Chase et al., 1993; Doyle et al., 1994); however, numerous questions remain. Some of the

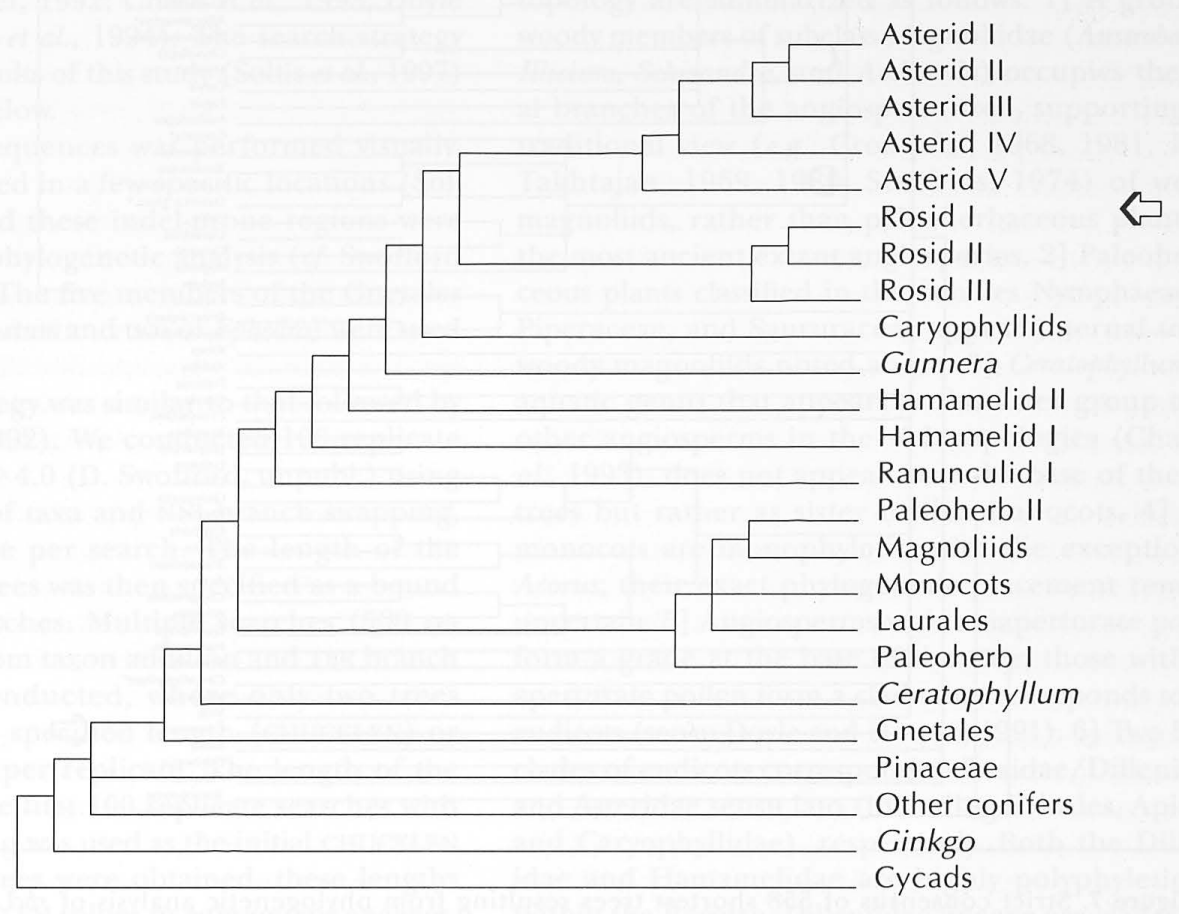

Figure 6. Summary of the major clades of angiosperms depicted in the strict consensus of 3,900 most parsimonious trees produced by analysis of $r b c \mathrm{~L}$ sequences for 499 species of seed plants (redrawn from Chase et al., 1993). All angiosperms with nodular nitrogen-fixing symbioses included in this analysis fall within the clade labeled Rosid I (note arrow). 


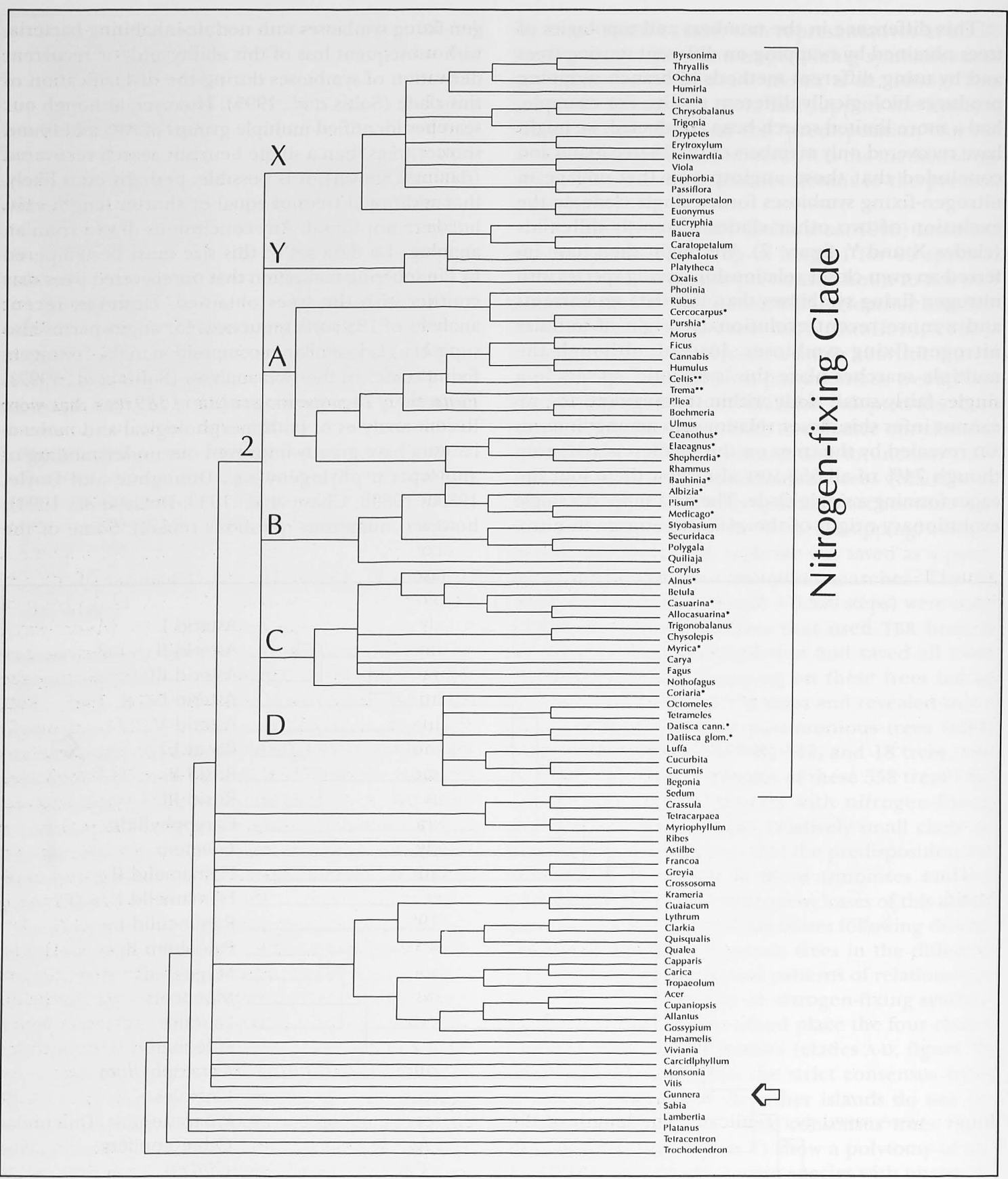

Figure 7. Strict consensus of 558 shortest trees resulting from phylogenetic analysis of $r b c \mathrm{~L}$ sequences for 99 species of angiosperms representing the Rosid I lineage and various outgroups. Representatives of families with nitrogen-fixing symbioses are indicated by asterisks. All angiosperm families with nodular nitrogen-fixing symbioses fall into a single clade ("Nitrogen-fixing Clade"), supported by a decay value of 2. Lineages labeled A-D are those clades within the Nitrogen-fixing Clade that actually contain the families involved in these symbioses; lineages X and $\mathrm{Y}$ do not contain any species with nitrogen-fixing symbioses. Lineages A-D form a single clade in all 18 trees found on the smallest island of most parsimonious trees and in $74 \%$ of the 558 trees found on all three islands. 
most notable perhaps are: 1] What are the most primitive extant angiosperms?; 2] What is the origin of the monocots?; and 3] What are the major groups of angiosperms and their interrelationships?

The phylogenetic analysis of $r b c \mathrm{~L}$ sequences for 499 species of seed plants (Chase et al., 1993) is the largest phylogenetic analysis of flowering plants conducted to date. Although many of the relationships supported by this analysis coincide with those inferred from traditional taxonomies, other relationships are anomalous and require further investigation. One possible test of these relationships is through corroboration by analysis of an independent data set (Miyamoto and Fitch, 1995). Because $r b c \mathrm{~L}$ is located in the chloroplast genome, corroboration of relationships by a nuclear-based tree would be most significant. To provide a nuclear-based phylogeny for the angiosperms for comparison with the $r b c \mathrm{~L}$ trees (Chase et al., 1993), we analyzed sequence variation in the $18 \mathrm{~S}$ ribosomal RNA genes (rDNA) in 223 species of angiosperms and five species of Gnetales (the closest extant relative of the angiosperms; Crane, 1985, 1988; Doyle and Donoghue, 1986, 1992; Loconte and Stevenson, 1990; Hamby and Zimmer, 1992; Chase et al., 1993; Doyle et al., 1994; Nixon et al., 1994). The search strategy and the general results of this study (Soltis et al., 1997) are summarized below.

Alignment of sequences was performed visually. Most indels occurred in a few specific locations (Soltis $e t$ al., 1997), and these indel-prone regions were omitted from the phylogenetic analysis ( $c f$. Swofford and Olsen, 1990). The five members of the Gnetales (three species of Gnetum and two of Ephedra) were used as outgroups.

The search strategy was similar to that followed by Maddison et al. (1992). We conducted 100 replicate searches using PAUP 4.0 (D. Swofford, unpubl.) using random addition of taxa and NNI branch swapping, saving a single tree per search. The length of the shortest of these trees was then specified as a bound in subsequent searches. Multiple searches (500 replicates) with random taxon addition and TBR branch swapping were conducted, where only two trees (NCHUCK $=2$ ) of a specified length (CHUCKLEN) or longer were saved per replicate. The length of the shortest trees in the first 100 replicate searches with NNI branch swapping was used as the initial CHUCKLEN value; as shorter trees were obtained, these lengths were used as CHUCKLEN values in subsequent analyses.

The 14 shortest trees obtained from these 500 replicate searches (ranging in length from 3,929 to 3,931 steps) were used as starting trees in searches using TBR branch swapping and saving all most parsmonious trees. Each of these 14 searches was allowed to run for several weeks on either a Macintosh Quadra 650 or a Power Macintosh 6100 computer; 2,000-5,000 trees were saved per search. After several months, each search had run for several weeks, but none had swapped to completion. The shortest trees obtained were of 3,923 steps, and trees of this length were obtained in two of the 14 searches. The strict consensus of the 5,294 trees saved from these two searches yielded a tree (figure 8) for comparison with the $r b c \mathrm{~L}$ topology (Chase et al., 1993).

Undoubtedly, shorter trees for the 18S rDNA data set exist, but the major lineages of angiosperms and their interrelationships appeared to have stabilized long before trees of length 3,923 were obtained. In fact, the same major lineages were obtained in trees more than 70 steps longer than the trees that were ultimately presented, although relationships among some of these lineages did not stabilize until much shorter trees were obtained.

The relationships supported by this analysis of $18 \mathrm{~S}$ rDNA sequences (figure 8; Soltis et al., 1997) are largely concordant with those inferred from $r b c \mathrm{~L}$ sequences (Chase et al., 1993). The major features of the 18S topology are summarized as follows. 1] A group of woody members of subclass Magnoliidae (Austrobaileya, Illicium, Schisandra, and Amborella) occupies the basal branches of the angiosperm tree, supporting the traditional view (e.g., Cronquist, 1968, 1981, 1988; Takhtajan, 1969, 1980; Stebbins, 1974) of woody magnoliids, rather than paleoherbaceous plants, as the most ancient extant angiosperms. 2] Paleoherbaceous plants classified in the families Nymphaeaceae, Piperaceae, and Saururaceae appear internal to the woody magnoliids noted above. 3] Ceratophyllum, an aquatic genus that appears as the sister group to all other angiosperms in the $r b c \mathrm{~L}$ topologies (Chase $e t$ al., 1993), does not appear near the base of the $18 \mathrm{~S}$ trees but rather as sister to the monocots. 4] The monocots are monophyletic, with the exception of Acorus; their exact phylogenetic placement remains uncertain. 5] Angiosperms with uniaperturate pollen form a grade at the base of the tree; those with triaperturate pollen form a clade that correponds to the eudicots (sensu Doyle and Hotton, 1991). 6] Two large clades of eudicots correspond to Rosidae/Dilleniidae and Asteridae sensu lato (including Ericales, Apiales, and Caryophyllidae), respectively. Both the Dilleniidae and Hamamelidae are highly polyphyletic. 7] Within the Rosidae/Dilleniidae lineage are clades that contain $i$ all the plants that produce glucosinolates (mustard oil glucosides) except Drypetes (Euphorbiaceae) and $i i]$ the "Saxifragales" (including Saxifragaceae sensu stricto (Soltis et al., 1993), Haloragaceae, Crassulaceae, Hamamelidaceae, Cercidiphyllaceae, Daph- 


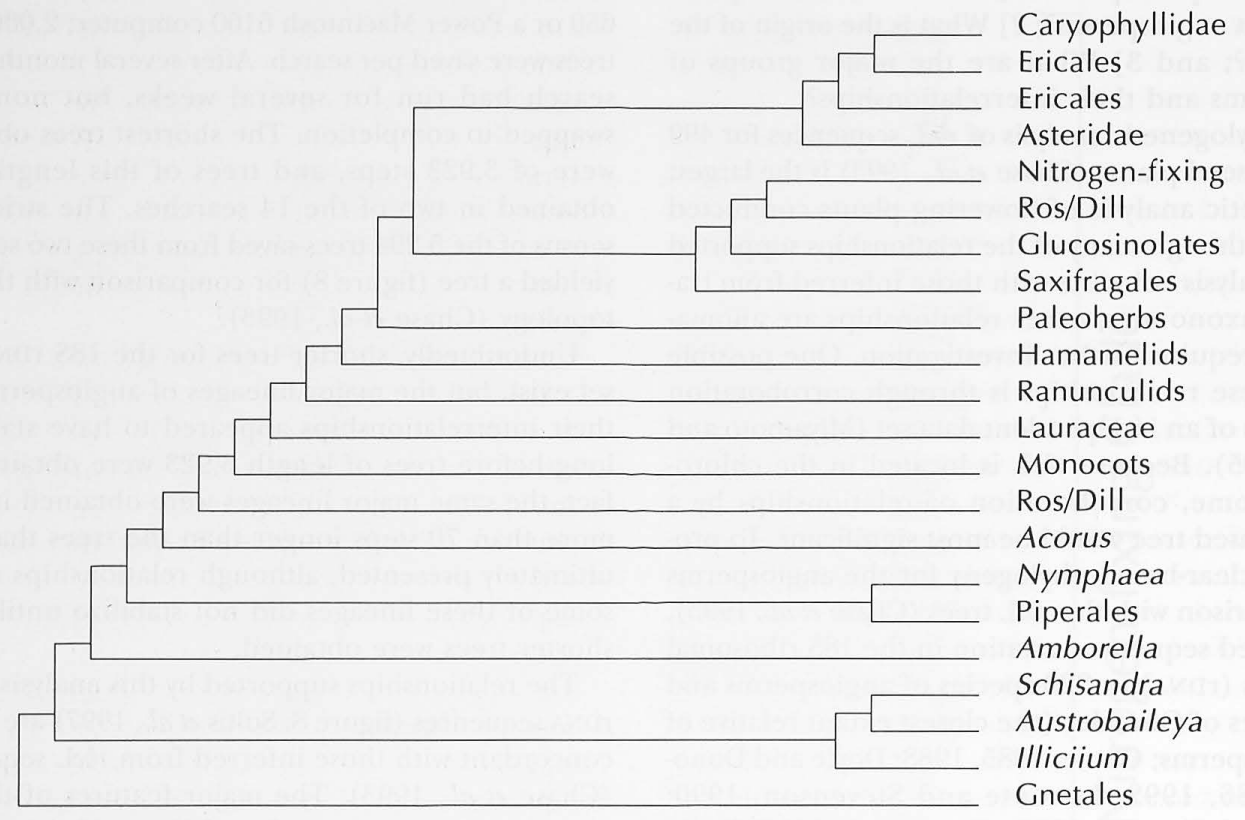

Figure 8. Summary of the major clades of angiosperms depicted in the strict consensus of 5,294 trees produced by analysis of $18 \mathrm{~S}$ rDNA sequences for 223 species of angiosperms and five species of Gnetales (Soltis et al., 1977) Lineages labelled Ros/Dill comprise genera of Rosidae and Dilleniidae.

niphyllaceae, and Paeoniaceae, families currently placed in three of Cronquist's (1981) six subclasses of dicots). 8] An expanded Caryophyllidae includes two families of carnivorous plants, Droseraceae and Nepenthaceae. These same lineages are also revealed by three other analyses of this same basic $18 \mathrm{~S}$ data set: one analysis identical to that described above plus two indel characters and two analyses of a slightly smaller data set (200 taxa) with and without indels. Most of these results also appear in the $r b c \mathrm{~L}$ topology; the only major conflicts between the $18 \mathrm{~S}$ and $r b c \mathrm{~L}$ trees are in the taxa occupying the basal branches of the tree, the position of the Caryophyllidae (but not its component families), and the uniaperturate grade of the $18 \mathrm{~S}$ tree versus the uniaperturate clade of the $r b c \mathrm{~L}$ tree.

Although the $r b c \mathrm{~L}$ analysis (Chase et al., 1993) did not find the shortest tree(s) for that data set (Rice $e t$ al., 1997) and the $18 \mathrm{~S}$ rDNA analysis did not swap to completion and thus did not likely find the shortest tree (s) (Soltis et al., 1997), it seems unlikely that both analyses, using data from different genes from different genomes, would converge on the same incorrect topology. The general congruence between the $18 \mathrm{~S}$ rDNA and $r b c \mathrm{~L}$ topologies provides strong support for several lineages (e.g., glucosinolate producers, Saxifragales, Asteridae sensu lato, Caryophyllidae sensu lato) and suggests that additional analyses using both more taxa and more genes should help to clarify major aspects of angiosperm phylogeny.

\section{Alternatives to large analyses: exemplars and data partitions}

Approximate searches of large data sets can also be conducted through other approaches. For example, a group of taxa known from other sources to form a clade could be represented in a large phylogenetic analysis by an "exemplar". This exemplar approach is really just taxon sampling designed to represent larger groups of taxa with one or a few taxa. For analyses designed to infer relationships among groups of well-recognized taxa, for which there is little disagreement over the circumscription of groups, the number of taxa actually included in the analysis could be only a small fraction of the original data set. Reducing the number of taxa in the analysis would permit more thorough searching for most parsimonious trees 
and could perhaps lead to topologies that would be unattainable with larger data sets.

The exemplar approach is not without problems of its own (see also Mishler, 1994). For example, how does one select an exemplar for a clade? Should the exemplar be a basal member or a derived member of the clade being sampled? Does the use of different exemplars for a clade affect the topology of the remainder of the tree? Does the use of a single exemplar for a group of related taxa affect the placement of either that clade or others on the tree? Morphological analyses of seed plant phylogeny (Doyle et al., 1994) clearly demonstrate that the selection of exemplars can affect resultant topologies (figure 9). For example, Doyle et al. (1994) tested the effects of selecting different genera to represent the angiosperms on the resulting seed plant phylogeny. Nine different angiosperm genera (e.g., from Magnoliales, Winteraceae, Piperales, Nymphaeales, Chloranthaceae, and others) were used singly and collectively to represent the angiosperms in this analysis. The use of single angiosperm taxa produced topologies that differed both in the position of the angiosperms within the anthophyte clade (angiosperms, Gnetales, Bennettitales, and Pentoxylon) and in that of the anthophyte clade within seed plants (figure 9). Based on these analyses, possible sister groups for the angiosperms include each of the other anthophyte lineages plus a group of Mesozoic seed ferns, but the real sister group of the angiosperms remains uncertain. The choice of exemplars is obviously not a trivial decision. Furthermore, the use of a single exemplar to represent a clade removes all close relatives of that exemplar from the analysis, both reducing the within-group variation and isolating the exemplar ( $c f$. Mishler, 1994). The concern then arises that an analysis will be reduced to a set of distantly related taxa, all with long branches. The undesirable topological effects of long and unequal branch lengths in parsimony analyses have been described theoretically (e.g., Felsenstein, 1978b) and demonstrated empirically (e.g., Olmstead et al., 1992) and in simulations (Huelsenbeck and Hillis, 1993). In fact, most empirical studies have called for more thorough sampling of groups to break up long branches by providing greater and equal taxon density throughout the tree (e.g., Olmstead et al., 1992; Chase et al., 1993). It is therefore ironic that an exemplar approach that both reduces taxon density and "creates" long and unequal branch lengths should be considered as an alternative to analysis of the entire data set. Were it not for the inefficiency of heuristic searches for large data sets, the use of exemplars would not seem an appealing alternative to more thorough analyses and would likely not receive serious consideration.
A second, and related but more attractive, alternative to analysis of an entire large data set involves the partitioning of taxa to permit analyses of subsets of the data, a general method of analysis referred to as "compartmentalization" (Mishler, 1994). Following this general approach, relationships within one or more subsets of taxa (inferred from previous analyses) are held constant in analyses of remaining groups. This approach derives, at least in part, from the "outgroup algorithm" of Maddison et al. (1984) for inferring ingroup relationships with various outgroups. As originally described (Maddison et al., 1984), the relationship between outgroup and ingroup taxa is constrained, as are relationships among multiple outgroups, to permit resolution of relationships within the ingroup. The ingroup itself could then be partitioned to permit separate analyses of its subsets. Although this method has not yet been widely used for analyses of molecular data sets for plants, a modification was used in several highly influential papers on seed plant and angiosperm phylogeny (e.g., Doyle, 1986; Donoghue and Doyle, 1989a, 1989b). The twostep procedure of Maddison et al. (1984), in which the position of the ingroup is established relative to the outgroups prior to analysis of ingroup relationships (with ingroup monophyly assumed), was followed in the reconstruction of angiosperm phylogeny based on morphological characters (Donoghue and Doyle, 1989a, 1989b). The placement of the angiosperms was first inferred from analyses that included all major lineages of seed plants, both fossil and extant (Doyle and Donoghue, 1986); the sister groups of the angiosperms were then used as outgroups in their subsequent analyses of angiosperm phylogeny (e.g., Donoghue and Doyle, 1989a, 1989b). The "outgroup algorithm" has been criticized on several grounds, most notably that of constrained ingroup monophyly and ingroup/outgroup relationships and the effects of these constraints on the overall topology (e.g., Nixon and Carpenter, 1993). Furthermore, the framework used in the second analysis is directly dependent on the scope, parameters, and procedures of the first; if the first part is flawed, then the second will likely be also. These criticisms may also apply to a partitioned analysis of the ingroup. As Doyle et al. (1994) note, however, conclusions from this type of analysis are not necessarily incorrect, even when alternative exemplars are used in the first step of the analysis. Experiments with different exemplars in analyses of seed plants have revealed little difference among angiosperm topologies in the subsequent analyses (Doyle et al., 1994).

Mishler (1994) provided a more explicit approach to compartmentalization that differs from both the exemplar approach and the outgroup algorithm 


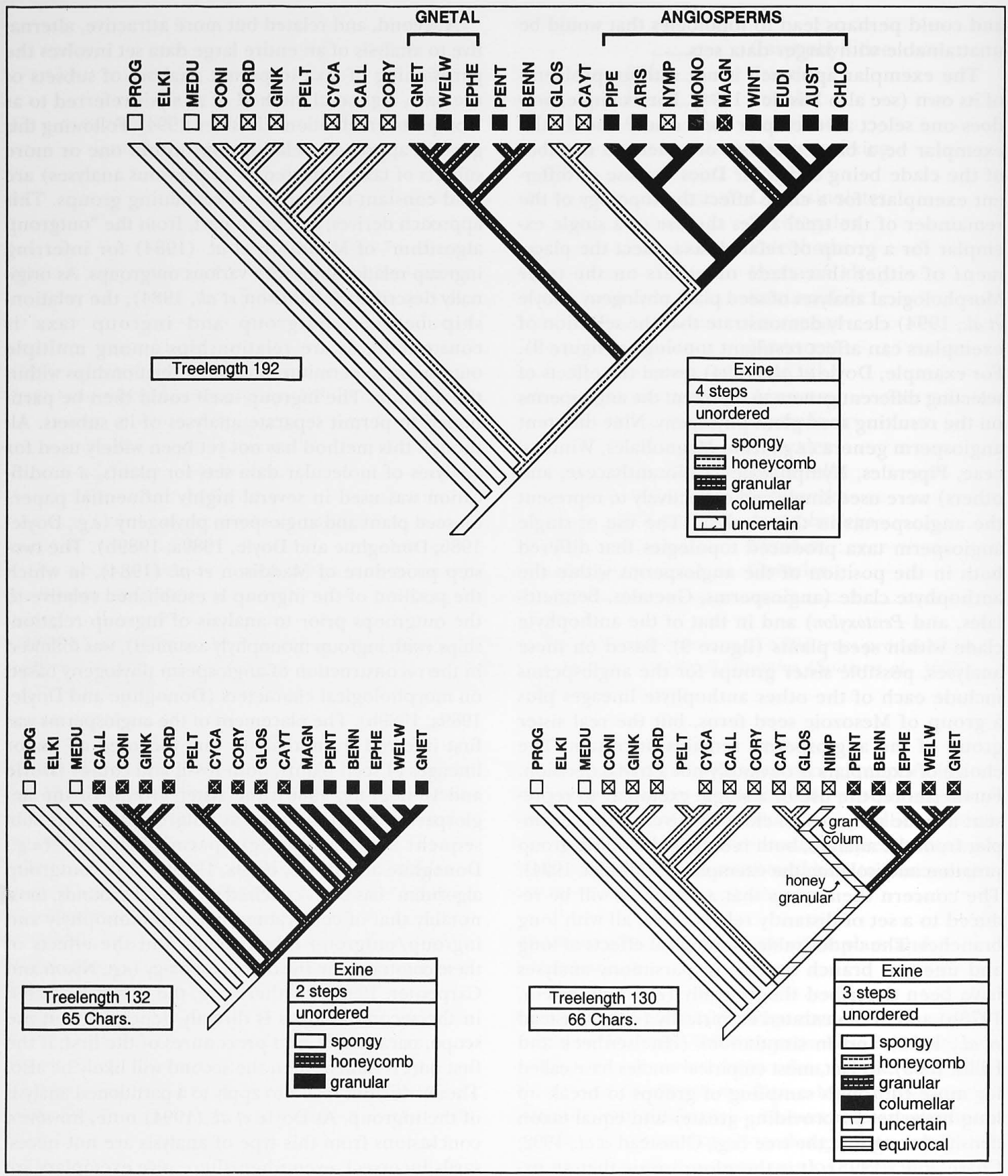

Figure 9. The topological effects of using different angiosperm genera as exemplars in analyses of seed plant phylogeny (from Doyle et al., 1994). Top: nine different angiosperm taxa or composite taxa (see text) were included with other seed plants. Bottom: a single taxon was used as an exemplar for the angiosperms in each of these analyses. Left, Magnoliales; right, Nymphaeales. Note topological differences among the three trees in both the position of the angiosperms within the anthophyte clade (angiosperms, Gnetales, Bennettitales, and Pentoxylon) and the position of the anthophyte clade within the seed plants. 
(Maddison et al., 1984) in that known monophyletic groups (i.e., compartments) are represented by an inferred hypothetical ancestor in more inclusive analyses. The character states of this ancestor are based on those of all the taxa that compose the monophyletic group, rather than a single exemplar, and the ancestor will likely differ from all real taxa in the group. The position of each monophyletic group (as represented by the hypothetical ancestor) is free to move relative to other taxa and groups in the analysis. Mishler (1994) recommended a three-step procedure: 1] a global analysis of all taxa to identify well-supported groups (i.e., compartments); 2] local analyses within compartments; and 3] further global analysis, with compartments represented by hypothetical ancestors or with compartment topologies constrained as found in local analyses. Of course, the most difficult aspect of a compartmentalized analysis such as this is the recognition of groups sufficiently well supported to be considered compartments. To provide reliable estimates of clades in a large data set, the global analysis in step (1) will likely require extensive computation, along the lines we recommend for analysis of an entire large data set (see above and figure 4). Perhaps the best approach to analyzing large data sets, therefore, is a thorough global analysis (e.g., per figure 4) to identify strongly-supported clades, followed by more thorough, local analyses of these clades and a subsequent analysis that examines relationships among these clades and the remaining taxa. To some extent, such analyses have already been conducted, but without the coordinated approach recommended by Mishler (1994). For example, the large $r b c \mathrm{~L}$ analysis of angiosperms (Chase et al., 1993) provided strong support for several clades, many of which have been the subject of additional, more focused studies (e.g., Onagraceae, Conti et al., 1993; glucosinolate producers, Rodman et al., 1993; Saxifragaceae sensu stricto, Morgan and Soltis, 1993; Soltis et al., 1993; Johnson and Soltis, 1994, 1995; Cornaceae, Xiang et al., 1993; Asteridae sensu lato, Olmstead et al., 1993; monocots, Duvall et al., 1993). Many of the latter studies used not only the $r b c \mathrm{~L}$ sequences of the original data matrix but also other characters for which homology assessments were clear within the compartment but would have been impossible across the angiosperms as a whole. Unfortunately, no effort has been made to date to conduct an additional global analysis using the information obtained from the local analyses of individual compartments. However, compartmentalization may represent a viable solution to the problems of large data sets, whether morphological, molecular, or a combination thereof.

\section{Confidence and prospectus}

Despite search strategies designed to explore large regions of tree space for large data sets and to find trees of shorter length than could be found in a single heuristic search, it is likely that additional trees of equal or shorter length exist but are not detected by the methods summarized in figure 4 . Thus, we are left with the following questions: 1] Has a local optimum been achieved? That is, has an analysis swapped to completion to yield one or more most parsimonious trees? 2] Does the local optimum represent the global optimum? That is, are there other, shorter trees that have not yet been recovered? In all likelihood, a local optimum achieved through analysis of a large data set is not the global optimum, and additional trees of shorter length have gone undetected. 3] Have all groups of most parsimonious trees been detected? Often we cannot answer these questions. However, in the phylogenetic analysis of $18 \mathrm{~S}$ rDNA sequences, we know with certainty that there are additional trees of the same length as those saved because the final set of searches did not swap to completion, and, in fact, there may be even shorter trees not yet recovered. However, we also know that the major lineages of angiosperms, as inferred from analysis of 18S rDNA sequences, are retained in trees many steps longer than the shortest trees recovered.

So, to what degree does one place confidence in relationships inferred from large analyses? Can large analyses in fact be used to infer relationships? We believe that large analyses can indeed be informative, but the degree of confidence that should be placed in such analyses will vary among studies. We suggest that the following criteria should be considered when conducting and evaluating analyses of large data sets: 1] the analysis attempted to explore large portions of tree space; 2] relationships are largely retained in trees much longer than the shortest trees; and 3] relationships are mostly congruent with those inferred from other data sets. We prefer the simultaneous resolution of relationships throughout the tree, if possible, to the separate resolution of subsets of the tree. Although the latter approach permits more thorough branch swapping and exploration of tree space for each component subset, it will not necessarily find a globally parsimonious solution that applies to the entire tree. However, when thorough analysis of an entire data set is not possible, compartmentalization appears to be a better alternative than the use of exemplars, an approach that is plagued with additional problems: how does one select appropriate longbranch exemplars? what effect does the replacement of a group of taxa with one or more exemplars have 
on the resultant topology? do the problems associated with inadequate taxon sampling (e.g., long-branch attraction, Felsenstein, 1978b) also arise when exemplars are substituted for groups of taxa? Clearly, these alternatives need to be evaluated theoretically and through comparative studies of real data, through simulations, or both.

Acknowledgments. This paper was initiated while the authors were Senior Mellon Fellows at the Smithsonian Institution's Laboratory of Molecular Systematics; we thank the Mellon Foundation and the LMS for their support and D. Swofford and E. Zimmer for helpful discussions. We also thank B. Mishler and M. Donoghue for helpful comments on the manuscript, E. De Luna and D. González for providing a Spanish translation of the Abstract, and J. Doyle, M. Donoghue, E. Zimmer, and the Annals of the Missouri Botanical Garden for permission to reprint figure 9.

\section{Literature cited}

Akkermans A.D.L., Van Dijk C. 1981. Non-leguminous root nodule symbioses with actinomycetes and Rhizobium. In: W. J. Broughton edr. Nitrogen Fixation. Vol. 1. Clarendon Press, Oxford, 57-103.

Al-Mallah M.K., Davey M.R., Cocking E.C. 1989. Formation of nodular structures on rice seedlings by Rhizobia. $J$. Exp. Bot. 40: 473-478.

Baker, Mullin B.C. 1992. Actinorhizal symbioses. In Stacey G., Burris R.H, Evans H.J. edrs. Biological Nitrogen Fixation. Chapman and Hall, New York, 259-291.

Bremer K. 1988. The limits of amino acid sequence data in angiosperm phylogenetic reconstruction. Evolution 42: 795-803.

Chase M.W., Soltis D.E., Olmstead R.G., Morgan D., Les D.H.,Mishler B.D., Duvall M.R., Price R.A., Hillis H.G., Qiu Y-L., Kron K.A., Rettig J.H., Conti E., Palmer J.D., Manhart J.R., Sytsma K.J., Michaels H.J., Kress W.J., Karol K.G., Clark W.D., Hedren M., Gaut B.S., Jansen R., Kim K.J., Wimpee C.F., Smith J.F., Furnier G.R., Strauss S.H., Xiang Q-Y., Plunkett G.M., Soltis P.S., Swensen S.M., Williams S.E., Gadek P.A., Quinn C.J., Eguiarte L.E., Golenberg E., Learn G.H. JR., Graham S.W., Barrett S.C.H., Dayanandan S., Albert V.A. 1993. Phylogenetics of seed plants: An analysis of nucleotide sequences from the plastid gene $r b c \mathrm{~L}$. Annals of the Missouri Botanical Garden 80: $528-580$.

Conti E., Fischbach A., Sytsma K.J. 1993. Tribal relationships in Onagraceae: Implications from $r b c \mathrm{~L}$ sequence data. Annals of Missouri Botanical Garden. 80: 672-685.

Crane P. 1985. Phylogenetic analysis of seed plants and the origin of the angiosperms. Annals of the Missouri Botanical Garden 72: 716-793.

Crane P. 1988. Major clades and relationships in the "higher" gymnosperms. In Beck CB edr. Origin and Evolution of Gymnosperms. Columbia Univ. Press, New York, 218-272.

Cronquist A. 1968. The Evolution and Classification of Flowering Plants. Houghton Mifflin, Boston.
Cronquist A. 1981. An Integrated System of Classification of Flowering Plants. Columbia Univ. Press, New York.

Cronquist A. 1988. The Evolution and Classification of Flowering Plants, 2nd ed. The New York Botanical Garden, New York.

Dahlgren R.T. 1980. A revised system of classification of the angiosperms. Botanical Journal Linnean Society 80: 91-124.

Donoghue M.J., Doyle J.A. 1989a. Phylogenetic analysis of angiosperms and the relationships of Hamamelidae. In Crane P.R., Blackmore S., edrs. Evolution, Systematics, and Fossil History of the Hamamelidae. Clarendon Press, Oxford, 17-45.

Donoghue M.J., Doyle J.A. 1989b. Phylogenetic studies of seed plants and angiosperms based on morphological characters. In: Fernholm B., Bremer K., Jornvall H., edrs. The Hierarchy of Life: Molecules and Morphology in Phylogenetic Analysis. Elsevier Science Publishers, Amsterdam, 181-193.

Donoghue M.J., Olmstead R.G., Smith J.F., Palmer J.D. 1992. Phylogenetic relationships of Dipsacales based on $r b c \mathrm{~L}$ sequences. Annals of the Missouri Botanical Garden 79: 333345.

Doyle J.A., Donoghue M.J. 1986. Seed plant phylogeny and the origin of the angiosperms: An experimental cladistic approach. Botanical Review 52: 321-431.

Doyle J.A., Donoghue M.J. 1992. Fossils and seed plant phylogeny reanalyzed. Brittonia 44: 89-106.

Doyle J.A., Donoghue M.J., Zimmer E.A. 1994. Integration of morphological and ribosomal RNA data on the origin of angiosperms. Annals of the Missouri Botanical Garden 81:419-450.

Doyle J.A., Donoghue M.J., Hotton C.L. 1991. Diversification of early angiosperm pollen in a cladistic context. In Blackmore S., Barnes S.H., edrs. Pollen and Spores: Patterns of Diversification. Clarendon Press, Oxford, 169-195.

Duvall M.R., Clegg M.T., Chase M.W., Clark W.D., Kress W.J., Hills H.G., Eguiarte L.E., Smith J.F., Gaut B.S., Zimmer E.A., Learn G.H., JR. 1993. Phylogenetic hypotheses for the monocotyledons constructed from $r b c \mathrm{~L}$ sequence data. Annals of the Missouri Botanical Garden 80: 607-619.

Felsenstein J. 1978a. The number of evolutionary trees. Systematic Zoology 27: 27-33.

Felsenstein J. 1978b. Cases in which parsimony or compatibility methods will be positively misleading. Systematic Zoology 27: 401-410.

Felsenstein J. 1981. Evolutionary trees from DNA sequences: A maximum likelihood approach. Journal of Molecular Evolution 17: 368-376.

Felsenstein J. 1985. Confidence limits on phylogenies: An approach using the bootstrap. Evolution 39: 783-791.

Hamby R.K., Zimmer E.A. 1992. Ribosomal RNA as a phylogenetic tool in plant systematics. In Soltis P.S., Soltis D.E., Doyle J.J., edrs. Molecular Systematics of Plants. Chapman and Hall, New York, 50-91.

Hendy M.D., Penny D. 1982. Branch and bound algorithms to determine minimal evolutionary trees. Mathemmatical Bioscience 59: 277-290.

Huelsenbeck J.P., Hillis D.M. 1993. Success of phylogenetic methods in the four-taxon case. Systematic Biology. 42: 247-264. 
Johnson L.A., Soltis D.E. 1994. matK DNA sequences and phylogenetic reconstruction in Saxifragaceae s. s. Sys tematic Botany 19: 143-156.

Johnson L.A., Soltis DE. 1995. Phylogenetic inference in Saxifragaceae sensu stricto and Gilia (Polemoniaceae) using matK sequences. Annals of the Missouri Botanical Garden 82: 149-175.

Loconte H., Stevenson D.W. 1990. Cladistics of the Spermatophyta. Brittonia 42: 197-211.

Maddison D.R. 1991. Discovery and importance of multiple islands of most-parsimonious trees. Systematic Zoology 40: 315-328.

Maddison D.R., Ruvelo M., Swofford D.L. 1992. Geographic origins of human mitochondrial DNA: Phylogenetic evidence from control region sequences. Systematic Biology 41: 111-124.

Maddison W.P., Donoghue M.J., Maddison D.R. 1984. Outgroup analysis and parsimony. Systematic Zoology 33: 83103.

Mishler B.D. 1994. Cladistic analysis of molecular and morphological data. American Journal of Physical Anthropology 94: 143-156.

Miyamoto M.M., Fitch W.M.. 1995. Testing species phylogenies and phylogenetic methods with congruence. Systematic Biology 44: 64-76.

Morgan D.R., Soltis D.E. 1993. Phylogenetic relationships among members of Saxifragaceae sensu lato based on $r b c \mathrm{~L}$ sequence data. Annals of the Missouri Botanical Garden 80: 631-660.

Mullin B.C. 1992. Symbiosis with Frankia. In Khush G.S., Bennett J., edrs. Nodulation and Nitrogen Fixation in Rice. International Rice Research Institute, Manila, The Phillipines, 67-75.

Mullin B.C., Swensen S.M., Goetting-Minesky P. 1990. Hypotheses for the evolution of actinorhizal symbioses. In Greshoff P.M., Roth L.E., Stacey G., Newton W.E., edrs. Nitrogen Fixation: Achievements and Objectives. Chapman and Hall, New York, 781-787.

Nixon K., Carpenter J. 1993. On outgroups. Cladistics 9: 413426.

Olmstead R.G., Michaels H.J., Scott K.M., Palmer J.D. 1992. Monophyly of the Asteridae and identification of their major lineages inferred from DNA sequences of $r b c \mathrm{~L}$. Annals of the Missouri Botanical Garden 79: 249-265.

Olmstead R.G., Bremer B., Scott K.M., Palmer J.D. 1993. A parsimony analysis of the Asteridae sensu lato based on rbcL sequences. Annals of the Missouri Botanical Garden 80: 700-722.
Rice K.A., Donoghue M.J., Olmstead R.G. 1997. A reanalysis of the large $r b c \mathrm{~L}$ data set. Implication for future phylogenetiic studies. Systematic Biology, in press.

Rodman J.E., Price R.A., Karol K., Conti E., Sytsma K.J., Palmer J.D. 1993. Nucleotide sequences of the $r b c \mathrm{~L}$ gene indicate monophyly of mustard oil plants. Annals of the Missouri Botanical Garden 80: 686-699.

Soltis D.E., Morgan D.R., Grable A., Soltis P.S., Kuzoff R.K. 1993. Molecular systematics of Saxifragaceae sensu stricto. American Journal of Botany 80: 1056-1081.

Soltis D.E., Soltis P.S., Morgan D.R., Swensen S.M., Mullin B.C., Dowd J.M., Martin P.G. 1995. Chloroplast gene sequence data suggest a single origin of the predisposition for symbiotic nitrogen fixation in angiosperms. Proceedings of the National Academy of Sciences USA 92: 2647-2651.

Soltis D.E., Soltis P.S., Nickrent D.L., Johnson L.A., Hahn W.J., Hoot S.B., Sweere J.A., Kuzoff R.K., Kron K.A., Chase M.W., Swensen S.M., Zimmer E.A., Chaw S-M., Gillespie L.J., Sytsma K.J.. 1997. Angiosperms phylogeny inferred from 185 ribosomal DNA sequences. Annals of the Missouri Botanical Garden 84:1-49

Sprent J.I., Raven J.R. 1992. In Stacey G., Burris R.H., Evans H.J., edrs. Biological Nitrogen Fixation. Chapman and Hall, New York, 461-496.

Stebbins G.L. 1974. Flowering Plants: Evolution above the Species Level. Belknap Press, Cambridge, Mass.

Swofford D.L. 1993. PAUP: Phylogenetic analysis using parsimony, version 3.1.1. Illinois Natural History Survey, Champaign.

Swofford D.L., Olsen G. 1990. Phylogeny reconstruction. In Hillis D.M., Moritz C., edrs. Molecular Systematics. Sinauer Associates, Sunderland, Mass., 411-501.

Takhtajan A. 1969. Flowering Plants: Origin and Dispersal. Oliver and Boyd, Edinburgh.

Takhtajan A. 1980. Outline of the classification of flowering plants (Magnoliophyta). Botanical Review 46: 225-359.

Thorne R. 1992. An updated classification of the flowering plants. Aliso 13: 365-389.

Torrey J.G., Berg R.H. 1988. Some morphological features for generic characterization among the Casuarinaceae. American Journal of Botany 75: 864-874.

Vigilant L., Stoneking M., Harpending H., Hawkes K., Wilson A.C. 1991. African populations and the evolution of human mitochondrial DNA. Science 253: 1503-1507.

Xiang Q-Y., Soltis D.E., Morgan D.R., Soltis P.S. 1993. Phylogenetic relationships of Cornus L. sensu lato and putative relatives inferred from $r b c \mathrm{~L}$ sequence data. Annals of the Missouri Botanical Garden 80: 723-734. 